\title{
Supporting Information The Interaction between Surface Acoustic Waves and Spin Waves: The Role of Anisotropy and Spatial Profiles of the Modes
}

\author{
Nandan K. P. Babu, ${ }^{\dagger}$ Aleksandra Trzaskowska, ${ }^{\dagger}$ Piotr Graczyk,$^{\dagger}$ \\ Grzegorz Centała, ${ }^{\dagger}$ Szymon Mieszczak, ${ }^{\dagger}$ Hubert Głowiński, ${ }^{\ddagger}$ Miłosz Zdunek, ${ }^{\dagger}$ \\ Stawomir Mielcarek, ${ }^{\dagger}$ and Jarosław. W. Kłos ${ }^{\dagger}$ \\ $\dagger$ Faculty of Physics, Adam Mickiewicz University, Poznań, Poland \\ $\ddagger$ Institute of Molecular Physics, Polish Academy of Sciences, Poznań, Poland
}

\section{Preparation of the sample and material parameters}

We used naturally oxidized (001) silicon as a substrate supporting the studied $\left[\mathrm{Co}_{20} \mathrm{Fe}_{60} \mathrm{~B}_{20} / \mathrm{Au}\right]_{20}$ multilayers deposited on top of a $4 \mathrm{~nm}$ titanium (Ti) and a $15 \mathrm{~nm}$ gold $(\mathrm{Au})$ buffer layers. The multilayers were deposited by magnetron sputtering in argon atmosphere $\left(\mathrm{P}_{A r}=1.4 \times 10^{-3}\right.$ mbar $)$ in a multi-chamber system with a base pressure below $2 \times 10^{-8}$ mbar. The thickness of each layer was controlled by the deposition time. ${ }^{1}$

The multilayer structure $\left(\left(\mathrm{Co}_{20} \mathrm{Fe}_{60} \mathrm{~B}_{20}(2.1 \mathrm{~nm}) / \mathrm{Au}(0.9 \mathrm{~nm})\right) \times 20\right)$ was designed to reduce the effective magnetization. According to Kittel's equation, by decreasing the effective magnetization it is possible to lower the resonance frequency in a given field. This means that the dispersion relation can be shifted by changing the effective magnetization. The effective magnetization is the sum of the shape anisotropy and the surface anisotropy. The shape anisotropy is defined by the saturation magnetization $\left(M_{S}=1200 \mathrm{kA} / \mathrm{m}\right)$ of the ferromagnetic layers and cannot be tuned without changing the alloy composition. On the other hand, the contribution of the surface anisotropy is inversely proportional to the thickness of the ferromagnetic layer. The $\mathrm{Co}_{20} \mathrm{Fe}_{60} \mathrm{~B}_{20} / \mathrm{Au}$ interface was found to have a surface anisotropy large enough to obtain an easy out-of-plane magnetization direction for films thinner than $1.1 \mathrm{~nm} .{ }^{1}$ By choosing a thickness of $2.1 \mathrm{~nm}$ of the $\mathrm{Co}_{20} \mathrm{Fe}_{60} \mathrm{~B}_{20}$ layer the surface anisotropy can be tuned to substantially reduce the effective magnetization and, as a result, lower the dispersion relation. We used a $0.9 \mathrm{~nm}$ thick Au spacer, which assured ferromagnetic coupling between layers. $^{2,3}$ So designed, the whole structure should behave like a single ferromagnetic film. 
Typically used for inducing surface anisotropy, heavy metals tend to increase the damping of the magnetic layers due to the spin pumping effect. Gold layers are an exception, since they are quite heavy and promote perpendicular anisotropy, but have low spin mixing conductance and long spin diffusion length. ${ }^{1}$ Using $\mathrm{Au}$ as a spacer we lower the effective magnetization without significantly increasing the damping.

By fitting the numerical data to the experimental results we determined the effective material parameters of the multilayer (treated as an effective medium). The magnetic parameters are: saturation magnetization $M_{S}=950 \mathrm{kA} / \mathrm{m}$ and exchange stiffness constant $A=5 \mathrm{pJ} / \mathrm{m}$. We assume the value of gyromagnetic ratio $\gamma=176 \mathrm{GHz} / \mathrm{T}$. The elastic parameters (the mass density $\rho$ and the components of the stiffness tensor c) of the constituent materials and the effective medium are specified in Tab. 1. The materials are assumed to be elastically isotropic.

Table 1: Elastic parameters of the constituent materials and the effective medium used in the model. We use the Voigt notation for the $\mathbf{c}$ tensor.

\begin{tabular}{|c||c|c|c|c|c|}
\hline & $\mathrm{Co}_{20} \mathrm{Fe}_{60} \mathrm{~B}_{20}$ & $\mathrm{Au}$ & $\mathrm{Ti}$ & $\mathrm{Si}$ & Effective layer \\
\hline \hline$\rho\left[\mathrm{kg} / \mathrm{m}^{3}\right]$ & 6970 & 19320 & 4500 & 2332 & 11535 \\
\hline$c_{11}(\mathrm{GPa})$ & 250 & 220 & 156 & 166 & 260 \\
\hline$c_{12}(\mathrm{GPa})$ & 100 & 160 & 67 & 64 & 176 \\
\hline$c_{44}(\mathrm{GPa})$ & 75 & 30 & 44.5 & 80 & 39 \\
\hline
\end{tabular}

The magnetoelastic coupling constant $b_{1}$ is determined using the strain modulated ferromagnetic resonance technique. ${ }^{4}$ The value obtained for the considered system is $b_{1}=-2.5 \mathrm{MJ} / \mathrm{m}^{3}$. We assume $b_{2}=b_{1}$.

\section{Experimental setup}

We studied the dispersion relations of thermally excited SAWs and magnetostatic SWs using a six-pass tandem Brillouin spectrometer (Scientific Instruments ${ }^{\complement}$ TFP2-HC - see Ref. 5), which ensures a contrast of $10^{15}$. A frequency-stabilized diode-pumped solid-state laser (Coherent ${ }^{\circledR}$ VERDI V5) operating at $\lambda_{0}=532 \mathrm{~nm}$ was used as a source of incident light. The measurements were performed in the $180^{\circ}$ backscattering geometry with crossed (p-s) polarization of incident and scattered light for SWs and non-crossed ( $p-p)$ polarization for SAWs. ${ }^{6,7}$ This BLS geometry setting allows collecting spectra for various configurations of the wave vector and the magnetic field. The backscattered light was collected by a lens with a $84 \mathrm{~mm}$ focal length and a 0.26 numerical aperture. A detailed description of the experimental setup, represented schematically in Figure 1, can be found in Ref 8. In our paper we present the experimental results obtained for two angles, $0^{\circ}$ and $45^{\circ}$, between 


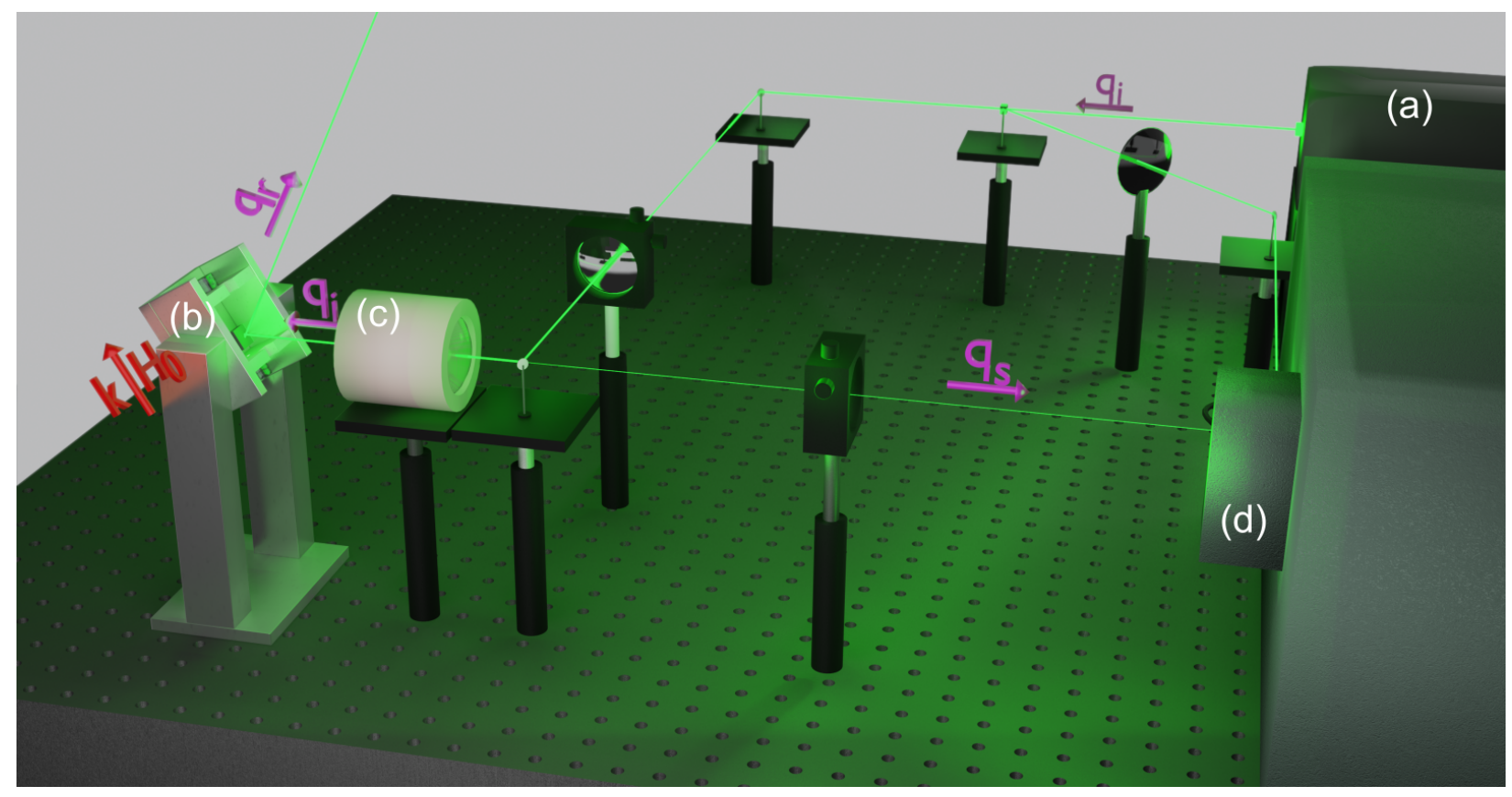

Figure 1: Schematic representation of the Brillouin light scattering (BLS) setup. The incident light beam is generated by a laser (Coherent ${ }^{\odot}$ VERDI V5) (a) and is directed to the sample, where the incident photons interact with magnons (SWs) and phonons (SAWs). The orientation of the sample holder (b) with respect to the direction of the incident beam determines the wave vector value $|\mathbf{k}|$ of the SWs and SAWs interacting with the light. Both the incident beam (of wave vector $\mathbf{q}_{i}$ ) and the backscattered beam $\left(\mathbf{q}_{s}\right)$ are shaped by an objective lens (c). The frequency spectrum of the backscattered beam is analyzed in a tandem Fabry-Perot interferometer (Scientific Instruments ${ }^{\circledR}$ TFP2-HC) (d). The refracted beam $\left(\mathbf{q}_{r}\right)$ is used to determine the orientation of the sample holder. A magnetic field $\mathbf{H}_{\mathbf{0}}$ is applied in the plane of the sample, parallel to $\mathbf{k}$.

the wave vector and the magnetic field. The Brillouin frequency shifts of the laser beam inelastically scattered from phonons and magnons are shown in Figure $4 .{ }^{9-11}$

In our experiment the wave vector value $k$ was varied from $0.6 \times 10^{5} \mathrm{~cm}^{-1}$ to $2.2 \times 10^{5} \mathrm{~cm}^{-1}$. The minimal accumulation time for each spectrum was 6000 cycles. In the paper we present phonon and magnon dispersion relations obtained for two magnetic field values, $30 \mathrm{mT}$ and $50 \mathrm{mT}$. By appropriately adjusting the magnitude of the applied magnetic field we can slightly shift up the SW dispersion relations in the frequency domain. This allows for the observation of magnetoelastic interactions in the dispersion relations of the F-SW, R-SAW and L-SAW modes in the wave vector range convenient for BLS measurements. The detailed behavior of magnons in the area of F-SW/L-SAW interaction is presented in Figure 4. The F-SW/L-SAW interaction is detectable only by the splitting of magnon peak because the phonon peak for L-SAW is barley measurable. The full width at half maximum (fwhm) of magnon peaks is small enough to trace the splitting with the charges of wave vector and plot 


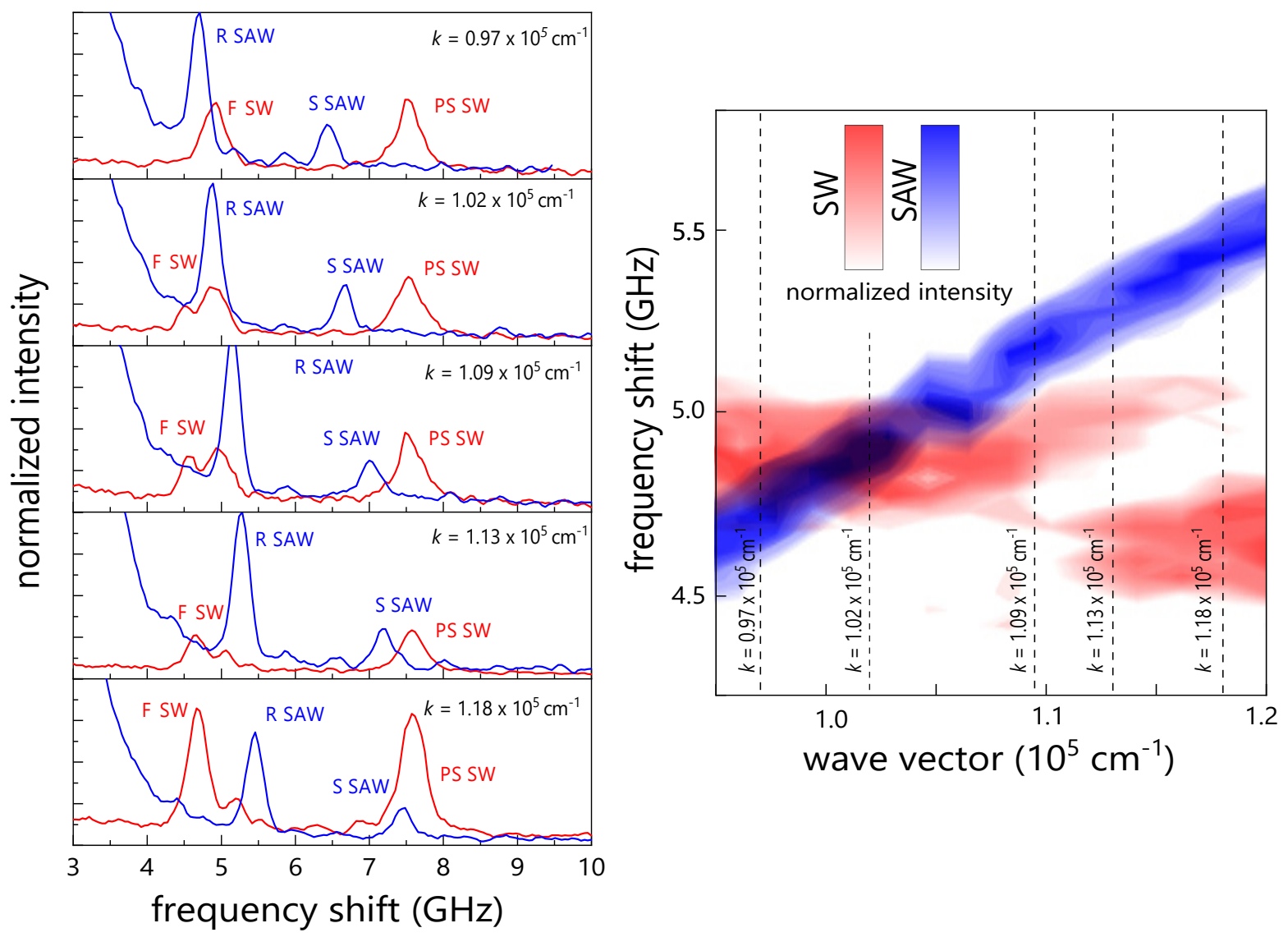

Figure 2: Anti-Stokes BLS spectra of magnons (red) and phonons (blue) measured in the considered system. The pairs of magnonic and phononic spectra, presented in each plot (in left column), were selected to show their evolution with the wave vector $k$, for fixed value of external field $\mu_{0} H=30 \mathrm{mT}$ and $\mathbf{H} \| \mathbf{k}$. The presented spectra include the peaks corresponding to the fundamental spin-wave (F-SW) mode, the first perpendicularly standing spin-wave (PS-SW) mode and Rayleigh SAW (R-SAW), Sezawa SAW (S-SAW). The Love SAW (L-SAW) of the frequency just below the frequency of R-SAW is practically invisible experimentally, except the region of interaction with F-SW $\left(k \approx 1.13 \times 10^{5} \mathrm{~cm}^{-1}\right)$, where it is detectable due the splitting of the F-SW peak. The right column presents the larger number of BLS spectra aggregated into dispersion relation, showing the F-SW/L-SAW anticrossig (i.e. interaction) and F-SW/R-SAW crossing (i.e. lack of interactions).

the F-SW/L-SAW anticrossing - see Figure 3.

\section{Theoretical model}

When we decompose the magnetization $\mathbf{M}$ and the magnetic effective field $\mathbf{H}_{\text {eff }}$ into static and dynamic parts: $\mathbf{M}(\mathbf{r}, t)=\mathbf{M}_{0}(\mathbf{r})+\mathbf{m}(\mathbf{r}, t), \mathbf{H}_{\mathrm{eff}}(\mathbf{r}, t)=\mathbf{H}_{0}(\mathbf{r})+\mathbf{h}(\mathbf{r}, t)+\mathbf{h}_{\mathrm{me}}(\mathbf{r}, t)$, where $\mathbf{h}_{\mathrm{me}}$ is the component of the dynamic field resulting from magnetoelastic interaction 


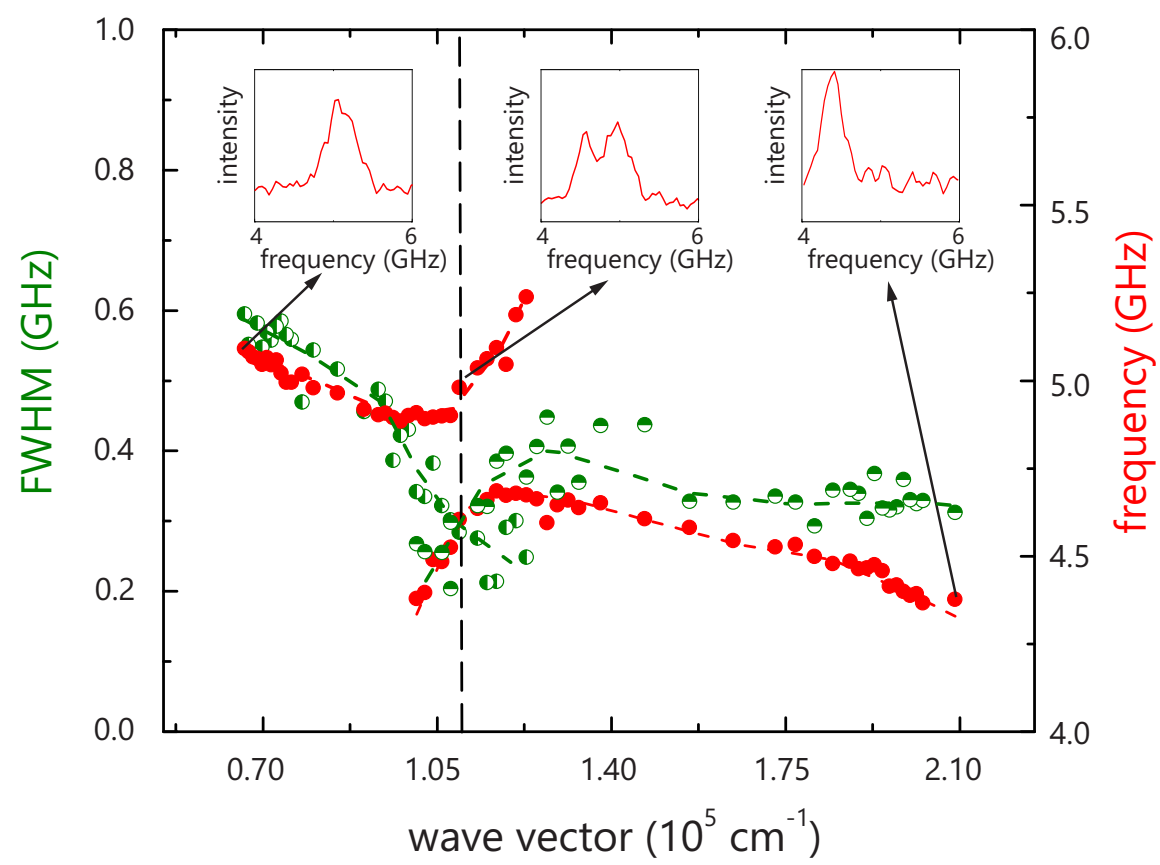

Figure 3: (a) The half-width-at-half-maximum (FWHM) of two peaks originating form F-SW mode (green/white points) in the region of interaction with L-SAW mode (vertical dashed line), in the dependence on the wave-vector value $k$, at the fixed value of magnetic field $\mu_{0} H=30 \mathrm{mT}$ and and $\mathbf{H} \| \mathbf{k}$. (b) The positions of the peaks (i.e. the frequencies of the splitted F-SW mode) are marked by the red points.

with SAWs, then the damping-free Landau-Lifshitz equation:

$$
\partial_{t} \mathbf{m}=-|\gamma| \mu_{0}\left(\mathbf{M} \times \mathbf{H}_{\mathrm{eff}}\right)
$$

can be written in the form:

$$
\partial_{t} \mathbf{m}=-|\gamma| \mu_{0}\left(\mathbf{M}_{0} \times\left(\mathbf{h}+\mathbf{h}_{\mathrm{me}}\right)-\mathbf{H}_{0} \times \mathbf{m}\right) .
$$

We assume that the external field $\mathbf{H}_{0}$ is applied in-plane, and, because of the homogeneity of the film, there are no static demagnetizing fields. Assuming further that the sample is saturated $\left(\mathbf{M}_{0} \| \mathbf{H}_{0}\right)$ and only considering the harmonic dynamics $\left(\mathbf{m}(\mathbf{r}, t)=\left[m_{\|}(\mathbf{r}), m_{\perp}(\mathbf{r}), 0\right] e^{i \omega t}\right.$, $\left.\mathbf{h}(\mathbf{r}, t)=\left[h_{\|}(\mathbf{r}), h_{\perp}(\mathbf{r}), 0\right] e^{i \omega t}, \mathbf{h}_{\mathrm{me}}(\mathbf{r}, t)=\left[h_{\mathrm{me}, \|}(\mathbf{r}), h_{\mathrm{me}, \perp}(\mathbf{r}), 0\right] e^{i \omega t}\right)$, we can obtain $\mathbf{M}_{0} \times \mathbf{H}_{0}$. With the non-linear terms neglected, $\mathbf{m} \times\left(\mathbf{h}+\mathbf{h}_{\text {me }}\right) \rightarrow 0$, the linearized Landau-Lifshitz equations for the in-plane $(\|)$ and out-of-plane $(\perp)$ components read:

$$
\left\{\begin{array}{rl}
i \omega m_{\|} & =|\gamma| \mu_{0}\left(-M_{S}\left(h_{\perp}+h_{\mathrm{me}, \perp}\right)+H_{0} m_{\perp}\right) \\
-i \omega m_{\perp} & =|\gamma| \mu_{0}\left(-M_{S}\left(h_{\|}+h_{\mathrm{me}, \|}\right)+H_{0} m_{\|}\right)
\end{array},\right.
$$


where $H_{0}=\left|\mathbf{H}_{0}\right|$, and $M_{S} \approx M_{0}=\left|\mathbf{M}_{0}\right|$ is the saturation magnetization. In the coordinate system presented in Figure 1 in the main paper $m_{x}=-m_{\|} \sin \phi, m_{y}=m_{\|} \cos \phi, m_{z}=m_{\perp}$, where $\phi$ is the deflection of the wave vector with respect to the direction of the external magnetic field.

Table 2: In-plane and out-of-plane components of the dynamic effective field of magnetoelastic origin induced by R/S-SAW or L-SAW for three selected angles $\phi$ between the in-plane applied field $\mathbf{H}_{0}$ and the wave vector $\mathbf{k}=\hat{\mathbf{x}} k$. Note that $\partial_{x} u_{x}=i k u_{x}, \partial_{x} u_{y}=i k u_{y}$, $\partial_{x} u_{z}=i k u_{z}$.

\begin{tabular}{|c|c|c|}
\hline \multicolumn{3}{|c|}{$\phi=0^{\circ}$} \\
\hline SAW & $\mathrm{R} / \mathrm{S}$ & $\mathrm{L}$ \\
\hline$\mu_{0} h_{\mathrm{me}, \|}$ & 0 & $\begin{aligned} & -2 b_{2} \varepsilon_{x y} \\
= & -i b_{2} k u_{y}\end{aligned}$ \\
\hline$\mu_{0} h_{\mathrm{me}, \perp}$ & $\begin{array}{c}2 b_{2} \varepsilon_{x z} \\
=b_{2}\left(\partial_{z} u_{x}+i k u_{z}\right) \\
\end{array}$ & 0 \\
\hline \multicolumn{3}{|c|}{$\phi=45^{\circ}$} \\
\hline SAW & $\mathrm{R} / \mathrm{S}$ & $\mathrm{L}$ \\
\hline$\mu_{0} h_{\mathrm{me}, \|}$ & $\begin{aligned} & b_{1} \varepsilon_{x x} \\
= & i b_{1} k u_{x}\end{aligned}$ & 0 \\
\hline$\mu_{0} h_{\mathrm{me}, \perp}$ & $\begin{aligned} & \sqrt{2} b_{2}\left(\varepsilon_{x z}+\varepsilon_{y z}\right) \\
&= \frac{\sqrt{2}}{2} b_{2}\left(\partial_{z} u_{x}+i k u_{z}\right) \\
&\end{aligned}$ & $\begin{aligned} & \sqrt{2} b_{2} \varepsilon_{y z} \\
&= \frac{\sqrt{2}}{2} b_{2} \partial_{z} u_{y} \\
&\end{aligned}$ \\
\hline \multicolumn{3}{|c|}{$\phi=90^{\circ}$} \\
\hline SAW & $\mathrm{R} / \mathrm{S}$ & $\mathrm{L}$ \\
\hline$\mu_{0} h_{\mathrm{me}, \|}$ & 0 & $\begin{aligned} & 2 b_{2} \varepsilon_{x y} \\
= & i b_{2} k u_{y}\end{aligned}$ \\
\hline$\mu_{0} h_{\mathrm{me}, \perp}$ & 0 & $\begin{aligned} & 2 b_{2} \varepsilon_{y z} \\
= & b_{2} \partial_{z} u_{y}\end{aligned}$ \\
\hline
\end{tabular}

The dynamic magnetic field components $h_{\|}$and $h_{\perp}$ are determined by the exchange and dipolar interactions between the dynamic components of magnetization, whereas the fields $h_{\mathrm{me}, \|}=\frac{1}{\mu_{0}} \frac{\partial F_{\mathrm{me}}}{\partial m_{\|}}$and $h_{\mathrm{me}, \perp}=\frac{1}{\mu_{0}} \frac{\partial F_{\mathrm{me}}}{\partial m_{\perp}}$ describe the impact of the strain generated by propagating SAWs on the magnetization dynamics: ${ }^{12}$

$$
\begin{aligned}
\mu_{0} h_{\mathrm{me}, \|} & =2 b_{2}\left(\varepsilon_{x z} \cos \phi+\varepsilon_{y z} \sin \phi\right) \\
\mu_{0} h_{\mathrm{me}, \perp} & =b_{1}\left(\varepsilon_{x x}-\varepsilon_{y y}\right) \sin 2 \phi-2 b_{2} \varepsilon_{x y} \cos 2 \phi
\end{aligned}
$$

and can be derived from the explicit form of the magnetoelastic energy density:

$$
F_{\mathrm{me}}=\frac{1}{M_{S}^{2}} \sum_{i, j=\{x, y, z\}} \epsilon_{i j} M_{i} M_{j}\left(b_{1} \delta_{i j}+b_{2}\left(1-\delta_{i j}\right)\right),
$$


where $\delta_{i j}$ is the Kronecker delta. The magnetization vector components are determined in the $x y z$ coordinate system: $M_{x}=M_{0} \cos \phi-m_{\|} \sin \phi, M_{y}=M_{0} \sin \phi+m_{\|} \cos \phi, M_{z}=m_{\perp}$.

In the considered geometry (with the external field $\mathbf{H}_{0}$ applied in the $x$ - $y$ plane and SAWs propagating along the $x$-direction, $\mathbf{k}=\hat{\mathbf{x}} k$ ) the strain tensor components figuring in eq. (4) are: $\varepsilon_{x x}=i k u_{x}, \varepsilon_{y y}=0, \varepsilon_{x y}=\frac{1}{2}\left(\partial_{y} u_{x}+\partial_{x} u_{y}\right), \varepsilon_{x z}=\frac{1}{2}\left(\partial_{z} u_{x}+i k u_{z}\right), \varepsilon_{y z}=\frac{1}{2} \partial_{z} u_{y}$. These formulas are further simplified for particular SAW types. For the R-SAW and S-SAW: $\varepsilon_{y y}=\varepsilon_{x y}=\varepsilon_{y z}=0$. The non-zero components are $\varepsilon_{x x}$ and $\varepsilon_{x z}$, with $\varepsilon_{x x}$ dominant for larger $k$. The $\varepsilon_{x z}$ component is reduced near the surface and therefore, in principle, has a smaller impact on the magnetoelastic coupling. For the L-SAW $\varepsilon_{x x}=\varepsilon_{y y}=\varepsilon_{x z}=0$. The only non-zero components of the strain tensor are $\varepsilon_{x y}$ and $\varepsilon_{y z} ; \varepsilon_{x y}>\varepsilon_{y z}$ close to the surface and for larger $k$.

Let us compare the $\mu_{0} h_{\mathrm{me}, \|}$ and $\mu_{0} h_{\mathrm{me}, \perp}$ values for the R/S-SAW and L-SAW for three selected orientations of the in-plane applied magnetic field: $\phi=0^{\circ}, 45^{\circ}$ and $90^{\circ}$. Table 1 presents the in-plane and out-of-plane components of the dynamic effective field of magnetoelastic origin, expressed by the respective strain tensor components, for these three orientations of the magnetic field.

If we consider the dominant strain tensor components that are maximal at the surface, $\varepsilon_{x y}$ and $\varepsilon_{x x}$, we notice that (i) the R/S-SAW drives the SW dynamics by the perpendicular component of the effective field, whereas the L-SAW affects SWs by the in-plane component of the effective field; (ii) the interaction is expected to be maximal with $\phi=0^{\circ}$ or $90^{\circ}$ for the L-SAW, and with $\phi=45^{\circ}$ for the R/S-SAW.

Let us discuss the reverse process, i.e. the influence of the SW dynamics on the SAWs. The fundamental elastodynamic equation, derived from Newton's $2^{\text {nd }}$ law of motion, reads:

$$
\rho \partial_{t^{2}} u_{x_{i}}=\partial_{x_{k}}\left(\sigma_{x_{i}, x_{k}}+\sigma_{\mathrm{me}, x_{i}, x_{k}}\right)
$$

with $\sigma_{\mathrm{me}, x_{i}, x_{k}}=\frac{\partial F_{\mathrm{me}}}{\partial \varepsilon_{x_{i}, x_{k}}}$ related to the respective derivatives of the magnetoelastic energy density component $F_{\mathrm{me}}$. Equation (6) can be written in the explicit form: ${ }^{13}$

$$
\begin{aligned}
\rho \partial_{t^{2}} u_{x} & =c_{44} \nabla^{2} u_{x}+\left(c_{44}+c_{12}\right) \partial_{x} \operatorname{div} \mathbf{u} \\
& +\underbrace{\frac{b_{1}}{M_{S}^{2}} \partial_{x}\left(M_{x}^{2}\right)+\frac{b_{2}}{M_{S}^{2}}\left(\partial_{y}\left(M_{x} M_{y}\right)+\partial_{z}\left(M_{x} M_{z}\right)\right)}_{\partial_{x_{k}} \sigma_{\mathrm{me}, x, x_{k}}},
\end{aligned}
$$




$$
\begin{aligned}
\rho \partial_{t^{2}} u_{y} & =c_{44} \nabla^{2} u_{y}+\left(c_{44}+c_{12}\right) \partial_{y} \operatorname{div} \mathbf{u} \\
& +\underbrace{\frac{b_{1}}{M_{S}^{2}} \partial_{y}\left(M_{y}^{2}\right)+\frac{b_{2}}{M_{S}^{2}}\left(\partial_{z}\left(M_{y} M_{z}\right)+\partial_{x}\left(M_{y} M_{x}\right)\right)}_{\partial_{x_{k}} \sigma_{\mathrm{me}, y, x_{k}}}, \\
\rho \partial_{t^{2}} u_{z} & =\underbrace{c_{44} \nabla^{2} u_{z}+\left(c_{44}+c_{12}\right) \partial_{z} \operatorname{div} \mathbf{u}}_{\partial_{x_{k}} \sigma_{\mathrm{me}, z, x_{k}}} \\
& +\underbrace{}_{\frac{1}{M_{S}^{2}} \partial_{y}\left(M_{z}^{2}\right)+\frac{b_{2}}{M_{S}^{2}}\left(\partial_{x}\left(M_{z} M_{x}\right)+\partial_{y}\left(M_{z} M_{y}\right)\right)} .
\end{aligned}
$$

In the linear approximation the spatial derivatives of magnetoelastic components of the stress tensor are equal and expressed by the formulas:

$$
\begin{aligned}
\partial_{x_{k}} \sigma_{\mathrm{me}, x, x_{k}} & =\frac{1}{M_{S}}\left(b_{2} \cos \phi \partial_{z} m_{\perp}-i k b_{1} \sin 2 \phi m_{\|}\right), \\
\partial_{x_{k}} \sigma_{\mathrm{me}, y, x_{k}} & =\frac{b_{2}}{M_{S}}\left(\sin \phi \partial_{z} m_{\perp}+i k\left(\cos ^{2} \phi m_{\|}+\sin ^{2} \phi m_{\perp}\right)\right), \\
\partial_{x_{k}} \sigma_{\mathrm{me}, z, x_{k}} & =\frac{b_{2}}{M_{S}} i k \cos \phi m_{\perp} .
\end{aligned}
$$

The terms $\partial_{x_{k}} \sigma_{\mathrm{me}, x, x_{k}}$ and $\partial_{x_{k}} \sigma_{\mathrm{me}, x, x_{k}}$ affect the dynamics of the displacement components $u_{x}$ and $u_{z}$. This allows for the determination of the impact of the R/S-SAW on SWs (described by $m_{\|}$and $m_{\perp}$ components). The term $\partial_{x_{k}} \sigma_{\text {me, } y, x_{k}}$ modifies the dynamics of the displacement component $u_{y}$, carrying the influence of the L-SAW on SWs. In Table 3 we summarize the formulas for $\partial_{x_{k}} \sigma_{\mathrm{me}, x_{j}, x_{k}}$ for three selected angles $\phi=0^{\circ}, 45^{\circ}$ and $90^{\circ}$.

If we consider waves with larger wave vectors (with $2 \pi / k$ much greater than the thickness of the magnetostrictive layer) and take into account the ellipticity of the SW (due to dynamic demagnetizing effects), $m_{\|}>m_{\perp}$, then we can notice that: (i) for $\phi=0$ the SW is coupled with the R/S-SAW (by $m_{\perp}$ ) and L-SAW (by $m_{\|}$), but its impact on the L-SAW should be more significant, since $m_{\|}>m_{\perp}$; (ii) for intermediate angles $\left(\phi=45^{\circ}\right) m_{\|}$carries the strongest influence of the SW on the R/S-SAW; its effect on the L-SAW is more difficult to analyze; it is definitely negligible for circular precession $\left(m_{\|}-m_{\perp}=0\right)$, observed in the absence of dipolar interaction, in which case the profile of the F-SW mode is uniform in amplitude $\left(\partial_{z} m_{\perp}\right)$, but for elliptical presession both terms $\left(m_{\|}-m_{\perp}\right.$ and $\left.\partial_{z} m_{\perp}\right)$ can cancel each other out, which is consistent with the lack of reverse coupling (i.e. the impact of the L-SAW on the SW, see Table 1); (iii) for $\phi=90^{\circ}$ the SW cannot affect the R/S-SAW, but can impact on the L-SAW by $m_{\perp}$ alone. 
Table 3: Spatial derivatives of the magnetoelastic contributions to the stress tensor, $\partial_{x_{k}} \sigma_{\mathrm{me}, x_{j}, x_{k}}\left(x_{j}=\{x, y, z\}\right)$, responsible for changes in the displacement $\left(u_{x}\right.$ and $u_{z}$ for $\mathrm{R} / \mathrm{S}-\mathrm{SAW}, u_{y}$ for L-SAW) induced by a SW (described by $m_{\|}$and $m_{\perp}$ components). We consider three angles $\phi$ between the in-plane applied external field $\mathbf{H}_{0}$ and the direction of the wave vector $\mathbf{k}=\hat{\mathbf{x}} k$. Note that $\partial_{x} m_{\perp}=i k m_{\perp}, \partial_{x} m_{\|}=i k m_{\|}$.

\begin{tabular}{|r|c|}
\hline \multicolumn{2}{|c|}{$\phi=0^{\circ}$} \\
\hline R/S-SAW,$u_{x}-\partial_{x_{k}} \sigma_{\mathrm{me}, x, x_{k}}$ & $\frac{b_{2}}{M_{S}} \partial_{z} m_{\perp}$ \\
\hline L-SAW,$u_{y}-\partial_{x_{k}} \sigma_{\mathrm{me}, y, x_{k}}$ & $\frac{b_{2}}{M_{S}} i k m_{\|}$ \\
\hline R/S-SAW,$u_{z}-\partial_{x_{k}} \sigma_{\mathrm{me}, z, x_{k}}$ & $\frac{b_{2}}{M_{S}} i k m_{\perp}$ \\
\hline \hline \multicolumn{2}{|c|}{$\phi=45^{\circ}$} \\
\hline R/S-SAW,$u_{x}-\partial_{x_{k}} \sigma_{\mathrm{me}, x, x_{k}}$ & $\frac{b_{2}}{M_{S}} \frac{\sqrt{2}}{2} \partial_{z} m_{\perp}-\frac{b_{1}}{M_{S}} i k m_{\|}$ \\
\hline L-SAW,$u_{y}-\partial_{x_{k}} \sigma_{\mathrm{me}, y, x_{k}}$ & $\frac{b_{2}}{M_{S}}\left(\frac{\sqrt{2}}{2} \partial_{z} m_{\perp}+\frac{1}{2} i k\left(m_{\|}-m_{\perp}\right)\right)$ \\
\hline R/S-SAW,$u_{z}-\partial_{x_{k}} \sigma_{\mathrm{me}, z, x_{k}}$ & $\frac{b_{2}}{M_{S}} \frac{\sqrt{2}}{2} i k m_{\perp}$ \\
\hline \hline \multicolumn{2}{|c|}{$\phi=90^{\circ}$} \\
\hline R/S-SAW,$u_{x}-\partial_{x_{k}} \sigma_{\mathrm{me}, x, x_{k}}$ & 0 \\
\hline L-SAW,$u_{y}-\partial_{x_{k}} \sigma_{\mathrm{me}, y, x_{k}}$ & $\frac{b_{2}}{M_{S}}\left(\partial_{z} m_{\perp}-i k m_{\perp}\right)$ \\
\hline R/S-SAW,$u_{z}-\partial_{x_{k}} \sigma_{\mathrm{me}, z, x_{k}}$ & 0 \\
\hline
\end{tabular}

\section{Numerical simulations}

Using the finite element method in COMSOL Multiphysics ${ }^{\complement} 14$ we solve numerically the coupled equations of motion for mechanic displacement and magnetization. The $\mathrm{CoFeB} / \mathrm{Au}$ multilayer is treated as a 60-nm-thick effective magnetic layer, and the $\mathrm{CoFeB} / \mathrm{Au}$ multilayer together with the Ti/Au buffer as an 80-nm-thick effective acoustic layer on a Si substrate. This is a reasonable assumption, since every single-layer thickness is much smaller than the wavelengths considered in this study.

The Landau-Lifshitz equation is implemented in the Mathematics module and solved (along with the Poisson equation for the magnetostatic potential) for the effective magnetic layer with the effective fields related to the exchange, dipolar, Zeeman and magnetoelastic interactions taken into account. The standard forms of effective fields are implemented. The magnetostrictive fields are obtained from the magnetostrictive energy density:

$$
\mu_{0} H_{\mathrm{me}, i}=\frac{2}{M_{S}^{2}} \sum_{j=\{x, y, z\}} \epsilon_{i j} M_{j}\left(b_{1} \delta_{i j}+b_{2}\left(1-\delta_{i, j}\right)\right) .
$$

The Landau-Lifshitz equation is coupled to the Solid Mechanics module solver for the acoustic waves by the magnetostrictive stress:

$$
\sigma_{\mathrm{me}, i j}=\frac{1}{M_{S}^{2}} M_{i} M_{j}\left(b_{1} \delta_{i j}+2 b_{2} \delta\left(1-\delta_{i j}\right)\right)
$$


The dispersion relations are obtained in the Eigenfrequency study. For this purpose the Floquet boundary conditions are implemented along the $x$ direction to define the wavevector $k$ in the model. No relaxation is necessary since the equilibrium static magnetization is always parallel to the external magnetic field in our system. To avoid noise in the solutions we set the scaling of all dependent variables to a fixed value.

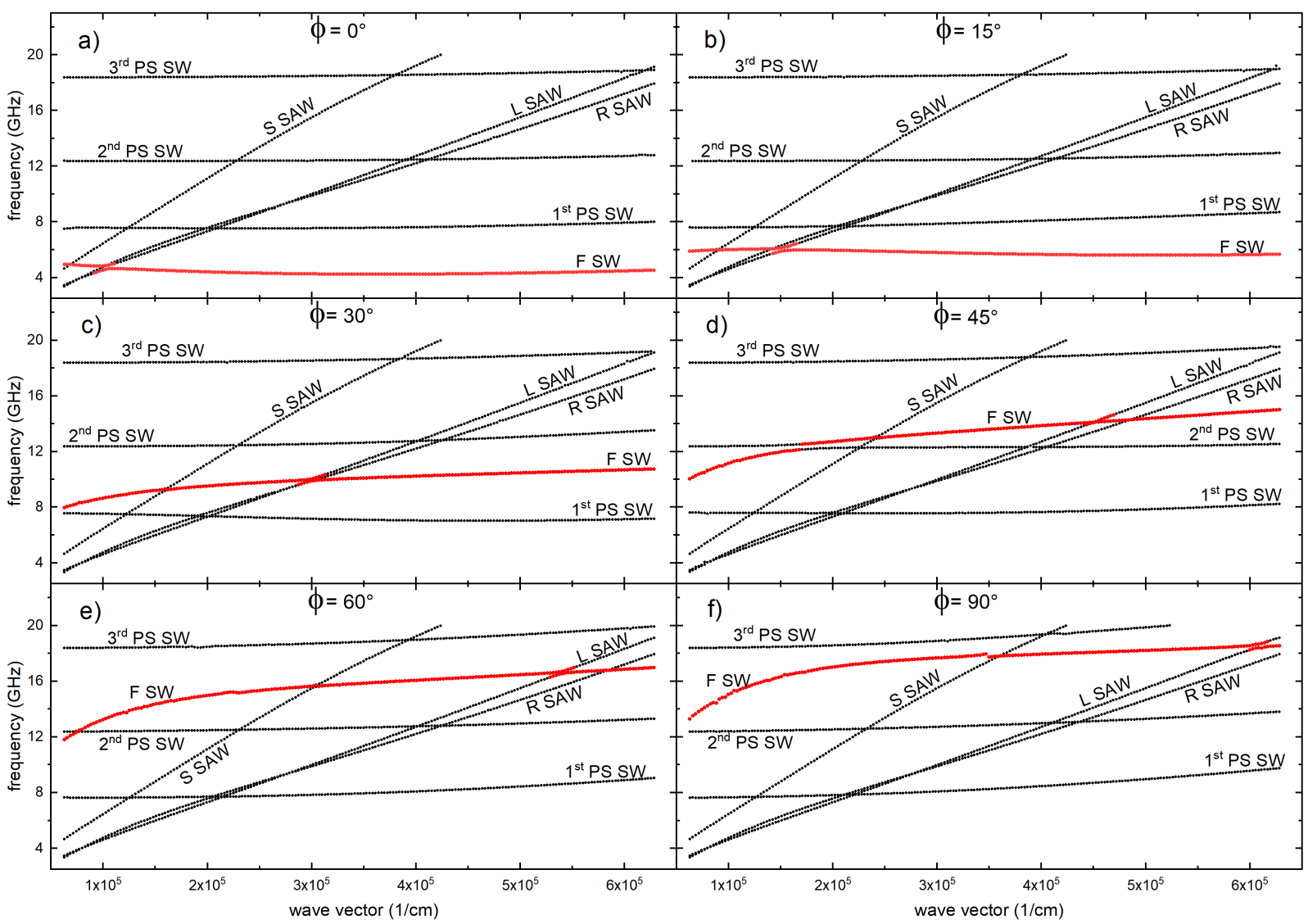

Figure 4: Magnetoelastic dispersion relations calculated for six angles between the external magnetic field $H_{0}$ and the wave vector $k$ : $\phi=0^{\circ}, 15^{\circ}, 30^{\circ}, 45^{\circ}, 60^{\circ}$ and $90^{\circ}$. The strongly anisotropic dispersion branch of the F-SW mode (red dots) changes position (frequencies) and slope (group velocity) with $\phi$. This results in angle-dependent positions of the (anti)crossings of the F-SW mode with the weakly anisotropic PS-SW mode.

To investigate the F-SW/SAW interaction for different angles $\phi$ between the external magnetic field $H_{0}$ and the wave vector $k$ we need to identify the F-SW mode in the rich magnetoelastic spectrum. We vary the angle $\phi$ from $0^{\circ}$ (backward volume configuration) to $90^{\circ}$ (Damon-Eshbach configuration); the dispersion relations calculated for $\phi=0^{\circ}, 15^{\circ}$, $30^{\circ}, 45^{\circ}, 60^{\circ}$ and $90^{\circ}$ are presented in Figure 4(a-f). As the angle $\phi$ increases, the fundamental mode (red points) shifts towards higher frequencies. As a result, the frequency and wave vector corresponding to the magnetoelastic coupling between the F-SW mode and the 
L-SAW rise as well. On the other hand, the strength of the F-SW/L-SAW magnetoelastic coupling reaches a global minimum at $45^{\circ}$ and a global maximum at $0^{\circ}$ in its angular dependence. It is worthy of notice that the identification of the F-SW mode requires the inspection of the profile of the SW modes (the F-SW mode should be uniform in phase across the magnetostrictive layer). This identification is vital in the vicinity of the F-SW/PS-SW (anti)crossings. At anticrossings the mixed character of the SW modes will affect the magnetoelastic interactions - see e.g. the kinks, resulting from the F-SW $/ 1^{\text {st }} \mathrm{PS}-\mathrm{SW}$ anticrossing, in the angular dependence of the F-SW/L-SAW frequency splitting $\Delta f$ at $19^{\circ}$ in Figure $4(\mathrm{a})$ in the main paper.

\section{References}

(1) Kuświk, P.; Głowiński, H.; Coy, E.; Dubowik, J.; Stobiecki, F. Perpendicularly magnetized Co20Fe60B20 layer sandwiched between Au with low Gilbert damping. J. Phys.: Cond. Matt. 2017, 29, 435803.

(2) Unguris, J.; Celotta, R. J.; Pierce, D. T. Determination of the Exchange Coupling Strengths for Fe/Au/Fe. Phys. Rev. Lett. 1997, 79, 2734-2737.

(3) Matczak, M.; Szymański, B.; Urbaniak, M.; Nowicki, M.; Głowiński, H.; Kuświk, P.; Schmidt, M.; Aleksiejew, J.; Dubowik, J.; Stobiecki, F. Antiferromagnetic magnetostatic coupling in $\mathrm{Co} / \mathrm{Au} / \mathrm{Co}$ films with perpendicular anisotropy. J. Appl. Phys. 2013, $114,093911$.

(4) Nesteruk, K.; Żuberek, R.; Piechota, S.; Gutowski, M. W.; Szymczak, H. Thin film's magnetostriction investigated by strain modulated ferromagnetic resonance at low temperature. Meas. Sci. Technol. 2014, 25, 075502.

(5) Scarponi, F. et al. High-Performance Versatile Setup for Simultaneous Brillouin-Raman Microspectroscopy. Phys. Rev. X 2017, 7, 031015.

(6) Carlotti, G. Elastic Characterization of Transparent and Opaque Films, Multilayers and Acoustic Resonators by Surface Brillouin Scattering: A Review. Appl. Sci. 2018, 8,124 .

(7) Mutti, P.; Bottani, C. E.; Ghislotti, G.; Beghi, M.; Briggs, G. A. D.; Sandercock, J. R. Advances in Acoustic Microscopy; Springer: Boston, 1995. 
(8) Trzaskowska, A.; Mielcarek, S.; Graczykowski, B.; Stobiecki, F. Surface waves investigation in $\mathrm{NiFe} / \mathrm{Au} / \mathrm{Co} / \mathrm{Au}$ multilayers by high-resolution Brillouin spectroscopy. $J$. Alloys Compd. 2012, 51\%, $132-138$.

(9) Sandercock, J. R. Light Scattering in Solids III: Recent Results; Springer: Berlin, 1982.

(10) Babu, N. K. P.; Trzaskowska, A.; Mielcarek, S.; Głowiński, H.; Chumak, O. M.; Zdunek, M.; Kłos, J. W.; Krawczyk, M. Interaction Between Thermal Magnons and Phonons in a CoFeB/Au Multilayer. IEEE Magn. Lett. 2019, 10, 1-5.

(11) Agrawal, M.; Vasyuchka, V. I.; Serga, A. A.; Karenowska, A. D.; Melkov, G. A.; Hillebrands, B. Direct Measurement of Magnon Temperature: New Insight into MagnonPhonon Coupling in Magnetic Insulators. Phys. Rev. Lett. 2013, 111, 107204.

(12) Dreher, L.; Weiler, M.; Pernpeintner, M.; Huebl, H.; Gross, R.; Brandt, M. S.; Goennenwein, S. T. B. Surface acoustic wave driven ferromagnetic resonance in nickel thin films: Theory and experiment. Phys. Rev. B 2012, 86, 134415.

(13) Gurevich, A.; Melkov, G. Magnetization Oscillations and Waves; CRC: Boca Raton, 1996.

(14) COMSOL Multiphysics Reference Manual, version 5.3", COMSOL, Inc. http:// comsol.com. 